\title{
Single top cross section measurements in the t-channel at CMS
}

\author{
Alberto Orso Maria Iorio* \\ Universitá degli studi di Napoli Federico II, \\ For the CMS collaboration \\ E-mail: oioriodcern.ch
}

The $t$-channel production cross section measurements in proton-proton collisions at the LHC with the CMS detector are presented here. Collision data at centre-of-mass energies of both 7 and 8 $\mathrm{TeV}$ collected during the years 2011 and 2012 are used. Three analyses are performed to extract the inclusive $t$-channel production cross section at $7 \mathrm{TeV}$ : a robust analysis making use of data driven techniques to extract the main backgrounds contributions and exploiting the characteristic pseudorapidity distribution of the recoil jet in signal events, and two multivariate analysis which take advantage of the full knowledge of the $t$-channel topology to increase the precision of the measurement. The first of the three analyses is extended to $8 \mathrm{TeV}$, measuring the inclusive cross section as well as the cross section for $t$-channel separate top and anti-top production. Constraints on the Cabibbo-Kobayashi-Maskawa matrix element $\mathrm{V}_{\mathrm{tb}}$ are extracted as well.

The European Physical Society Conference on High Energy Physics -EPS-HEP2013

18-24 July 2013

Stockholm, Sweden

${ }^{*}$ Speaker. 


\section{Introduction}

Single top quarks at the LHC can be produced through charged-current electroweak interactions. These processes are well suited to test the predictions of the standard model of particle physics and to search for new phenomena. Measurements of the single-top-quark production cross section also provide an unbiased determination of the Cabibbo-Kobayashi-Maskawa matrix element $\mathrm{V}_{\mathrm{tb}}$. Three types of production mechanisms are predicted by the standard model at the LHC: $t$-channel and $s$-channel processes, and $\mathrm{W}$-associated single-top-quark production (tW). The dominant contribution to the cross section is expected to come from the $t$-channel process with a cross section of $\sigma_{t-c h .}^{\text {th }}=64.6_{-0.7}^{+2.1}(\mathrm{scale})_{-1.7}^{+1.5}(\mathrm{pdf}) \mathrm{pb}[1]$ at $7 \mathrm{TeV}$, and of $\sigma_{t-\mathrm{ch} .}^{\mathrm{th}}=87.2_{-1.0}^{+2.8}(\mathrm{scale})_{-2.2}^{+2.0}$ (pdf) pb [2] at $8 \mathrm{TeV}$. Fig. 1 shows the Feynman diagram of single-top $t$-channel production. We present here the

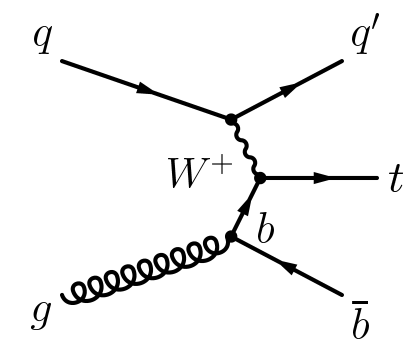

(a)

Figure 1: Leading order Feynman diagram for single-top production in the $t$-channel.

measurement of the $t$-channel cross sections with the CMS [3] experiment at 7 and $8 \mathrm{TeV}$.

\section{Analyses for cross section measurements}

The $t$-channel event signature (Fig. 1) typically comprises one forward jet scattered off a top quark, and a spectator b-jet from gluon splitting. The decay products of the top quark mainly appear in the central region of the detector. For the $7 \mathrm{TeV}$ CMS measurement [4], a dedicated event selection requiring one lepton, at least two jets, one of which b-tagged, is applied, and then measurements with two complementary approaches are performed. The first approach exploits the reconstructed top-quark mass and the pseudorapidity of the light jet recoiling against the top quark. This analysis is referred to as the $\eta_{j^{\prime}}$ analysis, and is designed to be straightforward, robust and have little model dependence. For this analysis a selection is performed requiring events with exactly 2 jets, exactly one of which b-tagged, and then requiring the reconstructed top mass to be inside a window around the nominal the top mass value [5]. A fit to the $\left|\eta_{j^{\prime}}\right|$ is performed on the surviving events. The second approach exploits, via multivariate discriminators, the compatibility of the signal candidates with the event characteristics predicted by the SM for electroweak top-quark production. This approach aims for a precise $t$-channel cross section measurement by optimising the discrimination between signal and background. The systematic uncertainties are constrained by simultaneously analysing phase space regions with substantial contributions from the $t$-channel single top and the backgrounds. For this reason those analyses selct events with 2-4 jets and 1-2 b-jets. Two independent multivariate analyses are performed with this approach, so to 
constitute a cross-check for each other, one based on a Neural Network (NN) discriminant and the other on Boosted Decision Trees (BDT) discriminant. To extract the signal a profile likelihood fit to the respective discriminant is used, where each background yield and most of the systematic uncertainties are treated as nuisance parameters. After verifying the consistency of the single results, the final value of the cross section is determined by combining the three analyses using the Best Linear Unbiased estimator (BLUE) [6]. The $\eta_{j^{\prime}}$ analysis is performed on the $8 \mathrm{TeV}$ data as well [7], slightly adapted to address the new conditions, introducing ad-hoc selection criteria to remove jets coming from pileup events and deriving the $\bar{t} \bar{t}$ background from orthogonal control samples in data.

\section{Results and conclusions}

The measured cross section values for 7 and $8 \mathrm{TeV}$ are:

$$
\begin{array}{cc}
\left.\left.\sigma_{\mathrm{t}-\mathrm{ch} ., 7 \mathrm{TeV}}=67.2 \pm 3.7 \text { (stat.) } \pm 4.6 \text { (syst. }\right) \pm 1.5 \text { (lumi. }\right) & (7 \mathrm{TeV}) \\
\left.\left.\sigma_{\mathrm{t}-\mathrm{ch} ., 8 \mathrm{TeV}}=80.1 \pm 5.7 \text { (stat.) } \pm 11.0 \text { (syst. }\right) \pm 4.0 \text { (lumi. }\right) & (8 \mathrm{TeV})
\end{array}
$$

Both those measurements are in good agreement with the standard model prediction. It is also possible to interpret this result in terms of $\mathrm{V}_{\mathrm{tb}}$ : under the assumption that $\left|\mathrm{V}_{\mathrm{td}}\right|,\left|\mathrm{V}_{\mathrm{ts}}\right|<<\left|\mathrm{V}_{\mathrm{tb}}\right|$, it yields $\left|\mathrm{V}_{\mathrm{tb}}\right|=\sqrt{\sigma_{\text {meas. }} / \sigma_{\text {theory }}}$. Taking into consideration only the most precise of the two measurements one finds:

$$
\left|\mathrm{V}_{\mathrm{tb}}\right|=1.026 \pm 0.046 \text { (meas.) } \pm 0.017 \text { (th.) } \quad(7 \mathrm{TeV}) .
$$

Assuming $\left|\mathrm{V}_{\mathrm{tb}}\right|<1$ one thus can retrieve a lower limit using the Feldman-Cousins [8] approach:

$$
0.92<\left|\mathrm{V}_{\mathrm{tb}}\right|<1 @ 95 \% \text { confidence level } \quad(7 \mathrm{TeV}) .
$$

Finally, it is possible also to extract a ratio between 8 and $7 \mathrm{TeV}$ cross sections, obtained taking only the $\eta_{j^{\prime}}$ component of the $7 \mathrm{TeV}$ analysis, getting as result $R_{8 / 7}=1.14 \pm 0.12$ (stat.) \pm 0.14 (syst.). The advantage is that part of the uncertainties is reduced or cancels out in the two measurements, thus potentially unvealing other deviations from the standard model behavior. Fig. 2 shows the measured cross sections as a function of the centre-of-mass energy. All measurements presented show a good agreement with the standard model prediction.

\section{References}

[1] Next-to-next-to-leading-order collinear and soft gluon corrections for t-channel single top quark production, N. Kidonakis, Phys. Rev. D 83, 091503(R)

[2] Differential and total cross sections for top pair and single top production, N. Kidonakis, arXiv: 1205.3453

[3] CMS Collaboration, "The CMS experiment at the CERN LHC", JINST 03 (2008) S08004.

[4] Measurement of the single-top-quark t-channel cross section in pp collisions at $\sqrt{s}=7 \mathrm{TeV}$, The CMS Collaboration, JHEP12(2012)035

[5] The Review of Particle Physics. J. Beringer et al. (Particle Data Group), Phys. Rev. D86, 010001 (2012) 


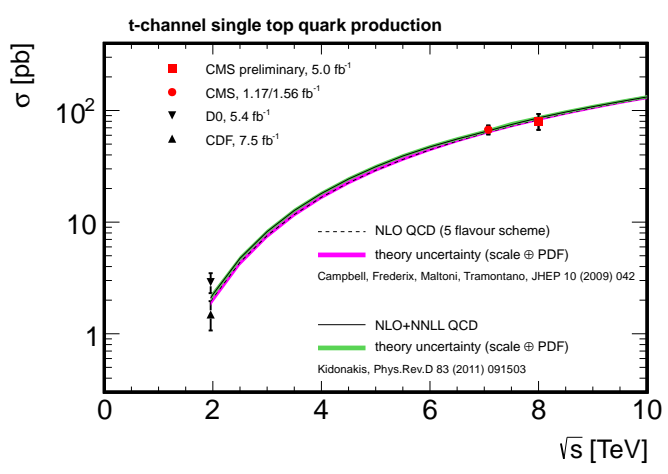

(a)

Figure 2: Summary of the single-top t-channel cross section measurements as function of the centre-ofmass energy.

[6] How to combine correlated estimates of a single physical quantity, Lyons, L. et al, Nucl. Instr. and Meth. A 270 (1988) 110.

[7] Measurement of the single top t-channel cross section in pp collisions at $\sqrt{s}=8 \mathrm{TeV}$, The CMS Collaboration, CMS PAS TOP-12-011

[8] A unified approach to the classical statistical analysis of small signals, Feldman, Gary J. and Cousins, Robert D., Phys. Rev. D 57 (1998) 3873, doi:10.1103/PhysRevD.57.3873, arXiv:physics/9711021. 